\title{
Estudo comparativo de casos entre a mobilização neural e um programa de alongamento muscular em lombálgicos crônicos
}

\author{
Comparative study of cases between neural mobilization and a muscular \\ elongation program on chronic backache
}

\author{
Guilherme Fortes Machado $^{[a]}$, Simone Eickhoff Bigolin ${ }^{[b]}$
}

[a] Fisioterapeuta graduado pela Universidade Regional do Noroeste do Estado do Rio Grande do Sul (UNIJUÍ), Ijuí, RS - Brasil, e-mail: guifisioterapia@bol.com.br

[b] Professora Mestre em Educação das Ciências, docente do curso de Fisioterapia da Universidade Regional do Noroeste do Estado do Rio Grande do Sul (UNIJUí), Ijuí, RS - Brasil, e-mail: simoneb@unijui.edu.br

\section{Resumo}

Introdução: A dor lombar, responsável por 50\% das disfunções músculo-esqueléticas, é uma das principais, senão a mais frequente, causa de dor, incapacidade funcional e laborativa. Objetivos: Avaliar os efeitos da mobilização neural e do alongamento na flexibilidade, no quadro álgico e nas atividades funcionais de sujeitos com dor lombar. Metodologia: Foram realizadas 20 sessões de um programa de mobilização neural e de um programa de alongamento muscular com dois grupos distintos em sujeitos que apresentavam lombalgia crônica, sendo estes divididos de forma aleatória. Como instrumentos para avaliação, foram utilizados a Escala Análogo-Visual da dor, a distância dedo-solo, a medida do ângulo de flexão do joelho e o questionário Roland Morris. Resultados: Foram avaliados nove sujeitos com dor lombar crônica; cinco deles participaram do grupo em que foi empregada a mobilização neural e quatro fizeram parte do grupo de alongamentos. Somente o programa de mobilização neural apresentou resultados significativos. Quando comparados os resultados dos programas não foi evidenciada diferença estatisticamente significativa nos critérios avaliados. Conclusão: Para esta amostra, os dois recursos terapêuticos apresentaram melhoras para as variáveis analisadas, porém apenas a mobilização neural demonstrou resultados significativos, não sendo possível, no entanto, apontar maior eficiência de um método em relação ao outro.

Palavras-chave: Dor lombar crônica. Mobilização neural. Alongamento muscular. 


\begin{abstract}
Introduction: The backache, responsible for 50\% of the skeletal muscle dysfunction, is one of the main otherwise the most recurrent cause of the pain, function incapacity and lobor. Objective: To analyze the effects of the neural mobilization and elongation in the flexibility on the neuralgia scene and in the functional activities of people with back pain. Methodology: Twenty sessions were fulfilled of a neural mobilization program and of a muscular elongation program with two distinct groups of people who showed chronic backache, divided in aleatory form. As instruments for analysis it was used the Analog-Visual of the pain Scale, the the distance finger-ground, the measurement angle of the knee flexion and the Roland Morris questionnaire. Results: Nine people were analyzed with chronic back pain; five of them attended the group wherein the neural mobilization was used and four (4) were part of elongation group. Only the neural mobilization program showed significant effects. When the results of the programs were compared, no significant statistically difference was evident in the criterion estimated. Conclusion: For this sample, both therapeutic resources showed recovery to the variable analyzed, however only the neural mobilization showed significant results, being impossible, however, point out more efficiency of one method in respect to the other.
\end{abstract}

Keywords: Chronic back pain. Neural mobilization. Muscular elongation.

\title{
Introdução
}

Lombalgia é a definição dada a todas as categorias de dor com ou sem rigidez, que se localizem na região inferior do dorso entre o último arco costal e a prega glútea, ocorrendo normalmente na linha média, na região correspondente à quarta e à quinta vértebra lombar (L4-5) (1). Estudos indicam que a dor lombar é responsável por 50\% das disfunções músculo-esqueléticas e uma das principais, senão a mais frequente, causa de dor, incapacidade funcional e laborativa de sujeitos da faixa etária compreendida entre 30 e 45 anos e considerada a terceira causa de incapacidade para qualquer faixa etária (2).

No Brasil existem estimativas de que mais de 10 milhões de pessoas sofram com a incapacidade relacionada à dor lombar (3). A perspectiva é de que cerca $70 \%$ a $80 \%$ da população sofrerá um episódio de dor na vida, tornando-se uma das patologias mais encontradas na prática fisioterapêutica (4).

São conhecidas inúmeras patologias capazes de se manifestar como lombalgia, e é consenso que uma significativa parcela dos indivíduos acometidos não possui um diagnóstico preciso quanto à causa da dor, pois há que se considerar o grande número de estruturas da coluna que podem ser fontes de dor (5). Para Mendes (6), os principais fatores responsáveis pelas dores lombares são problemas psicológicos, baixo nível de escolaridade, atividade pesada ou em postura sentada, levantar grandes quantidades de peso, sedentarismo, acidentes de trabalho, dirigir veículos, horas excessivas de trabalho, gravidez, ferimentos, tabagismo, entre outros.

A literatura, no entanto, mostra que a dor nas costas é inespecífica em $85 \%$ dos casos, uma vez que, sendo cada tecido inervado, qualquer um desses tecidos pode ser responsável pela dor; consequentemente todos eles podem ser fonte de dor: discos, ligamentos, articulação sacroilíaca, músculos e estruturas nervosas (7).

Por sua relevância, diferentes estudos sobre dor lombar vêm sendo desenvolvidos com o objetivo de abolir e/ou controlar o sintoma, empregando uma gama variada de técnicas, algumas delas ainda em processo de validação (8). Na tentativa de encontrar alternativas terapêuticas eficazes no tratamento da dor lombar, este estudo buscou avaliar os efeitos de dois programas terapêuticos diferentes a serem aplicados para a recuperação e minimização dos sintomas lombares.

Assim, foram analisados os efeitos de um programa de Mobilização Neural (MN) e de um programa de alongamento muscular em sujeitos que apresentavam lombalgia crônica. Cabe salientar que foram investigados os efeitos das referidas técnicas direto na dor, na flexibilidade e na realização das atividades funcionais. Nesse ponto é importante referir que a mobilização neural é uma técnica antiga, porém pouco utilizada, que procura restaurar o movimento e a elasticidade da estrutura afetada, promovendo o retorno às suas funções normais. Muitos estudos já demonstraram a grande eficácia da mobilização neural para o tratamento e prevenção de determinadas patologias. 
A mobilização neural pode ser conceituada como um conjunto de técnicas que tem como objetivo impor ao sistema nervoso maior tensão, mediante determinadas posturas para que, em seguida, sejam aplicados movimentos lentos e rítmicos direcionados aos nervos periféricos e à medula espinhal, proporcionando melhora na condutibilidade do impulso nervoso (9).

Já o alongamento muscular é reconhecidamente eficaz no tratamento das mais diversas disfunções do sistema músculo-esquelético, sendo uma das melhores técnicas para aumento da flexibilidade e, até então, a conduta terapêutica mais frequentemente adotada na Fisioterapia. Cabe lembrar que o alongamento é uma manobra terapêutica empregada para aumentar o comprimento de tecidos moles que estejam encurtados, podendo ser definido também como técnica aplicada para aumentar a extensibilidade músculo-tendinosa e do tecido conjuntivo periarticular, contribuindo assim para aumentar a flexibilidade articular (10).

Como até o presente momento não se conhece muitos estudos publicados que comprovam a eficiência da mobilização neural para a redução da dor e aumento da flexibilidade, torna-se pertinente a realização de trabalhos que possam demonstrar estes benefícios por meio da técnica mencionada.

\section{Materiais e métodos}

O presente estudo caracteriza-se por ser do tipo experimental com abordagem quantitativa sob seleção elegível. Foi aprovado pelo Comitê de Ética em Pesquisa da Universidade Regional do Noroeste do Estado do Rio Grande do Sul (Unijuí), mediante Parecer Consubstanciado n. 040/2008.

A amostra foi composta por dez indivíduos que faziam parte da lista de espera da Clínica-Escola de Fisioterapia da Unijuí, de ambos os gêneros. Os participantes foram divididos em dois grupos, cada um com cinco integrantes, sendo esta divisão realizada de maneira aleatória, ou seja, não houve critérios específicos para esta divisão. Um grupo foi submetido a um programa de mobilização neural (Grupo 1), sendo empregado protocolo específico para este grupo, composto por quatro exercícios. Primeiramente foi realizado o SLR e posteriormente três exercícios descritos por Mulligan, que objetivam pôr o sistema nervoso em tensão. O outro grupo foi submetido a um programa de alongamentos musculares (Grupo 2) ativos e passivos que trabalhavam os músculos extensores do tronco, flexores laterais, latíssimo, rotadores do tronco, glúteos, rotadores externos e internos do quadril, adutores, abdutores, extensores e abdutores do quadril, flexores e extensores do joelho e gastrocnêmico e sóleo. Todos os indivíduos da pesquisa eram portadores de lombalgia crônica (dor lombar por mais de três meses), residentes no município de Ijuí, RS.

Como critérios de exclusão, levou-se em conta a presença de patologias inflamatórias agudas da coluna lombar, fratura de coluna, cirurgia prévia de coluna, qualquer seqüela que cause limitação na ADM (amplitude de movimento) de membro inferior, indivíduos que apresentem precaução para mobilização neural como: distúrbios neurológicos; patologia sistêmica, imunodepressão, portadores de câncer, distúrbios psicológicos, distúrbios cognitivos e impossibilidade, por qualquer motivo, de comparecer à Clínica-Escola pelo menos duas vezes por semana para a participação na presente pesquisa.

Como instrumentos para a coleta de dados utilizou-se:

- teste de Slump: para avaliação da tensão neural;

- flexímetro para avaliação da flexibilidade da cadeia muscular posterior, mensurando o ângulo de flexão do joelho;

- distância dedo-solo;

- questionário Rolland-Morris para avaliação das atividades físico-funcionais;

- Escala Análogo-Visual (EAV) para analisar o comportamento do quadro álgico na $1^{\mathrm{a}}, 5^{\mathrm{a}}, 10^{\mathrm{a}}, 15^{\mathrm{a}}$ e $20^{a}$ sessão.

As intervenções foram realizadas no mínimo duas vezes por semana, com duração de aproximadamente 30 minutos cada, totalizando de 20 sessões. Ao fim dos programas terapêuticos, os sujeitos foram reavaliados pelos mesmos instrumentos utilizados inicialmente. 
Os resultados da pesquisa foram analisados pela estatística descritiva utilizando média e desviopadrão. Com o intuito de realizar uma análise estatística mais aprofundada foi utilizado o Teste de Wilcoxon, para avaliar os resultados pré e pós entre os indivíduos do mesmo grupo, e o teste de Mann-Whitney, para a análise comparativa entre os grupos do estudo, ambos considerando significativos quando $\mathrm{p} \leq 0,05$.

\section{Resultados}

Inicialmente destaca-se que as avaliações foram realizadas com dez sujeitos. No transcorrer do programa houve a desistência de um participante, o que fez com que os resultados obtidos com este sujeito fossem excluídos da análise final. Assim, nove sujeitos deram continuidade ao tratamento, sendo que sete são do gênero feminino e dois do gênero masculino, com idade média de 44,22 \pm 8,54 anos.

No teste de Slump, seis sujeitos apresentaram tensão neural, dos quais cinco foram integrantes do Grupo 1 e apenas um do Grupo 2.

Depois de realizadas as 20 sessões de intervenção e reavaliados os participantes, apenas um manteve a resposta positiva ao teste, o qual participou do programa de mobilização neural, tendo os demais apresentado resultados negativos.

A avaliação inicial da dor pela EAV realizada no Grupo 1 apresentou média de 5,6 \pm 2,50, com uma redução do quadro álgico pós tratamento para 1,6 \pm 2,07, sendo esta alteração estatisticamente significativa $(\mathrm{p} \leq 0,039)$. É importante ressaltar que dois tiveram abolidas suas dores ao fim do programa de mobilização neural.

No que se refere ainda ao quadro álgico, o Grupo 2 apresentou uma avaliação média de 3,5 \pm 1,91 pontos na EAV no período pré-tratamento, passando para $0,5 \pm 1$, apresentando, desta forma, redução do quadro álgico. Ao ser realizada análise estatística, no entanto, esta redução não pôde ser considerada significativa para esta amostra $(\mathrm{p} \leq 0,063)$. Deve ser levado em consideração, porém, o fato de três pacientes também terem abolidas as suas dores.

Comparando os valores pós-intervenção, e as diferenças entre as médias pré e pós-intervenção, não houve diferença estatisticamente significativa para esta amostra ( $\mathrm{p} \leq 0,345)$, ou seja, embora o programa de mobilização neural resultasse em melhora significativa para a redução do quadro álgico, não foi possível, para esta amostra, identificar a maior eficácia do programa de tratamento em relação ao alongamento muscular.

É preciso considerar, contudo, que ambos os protocolos resultaram em melhoras para os sujeitos avaliados, como ilustrado abaixo no Gráfico 1; no entanto, somente o programa de mobilização neural mostrou grau de significância.

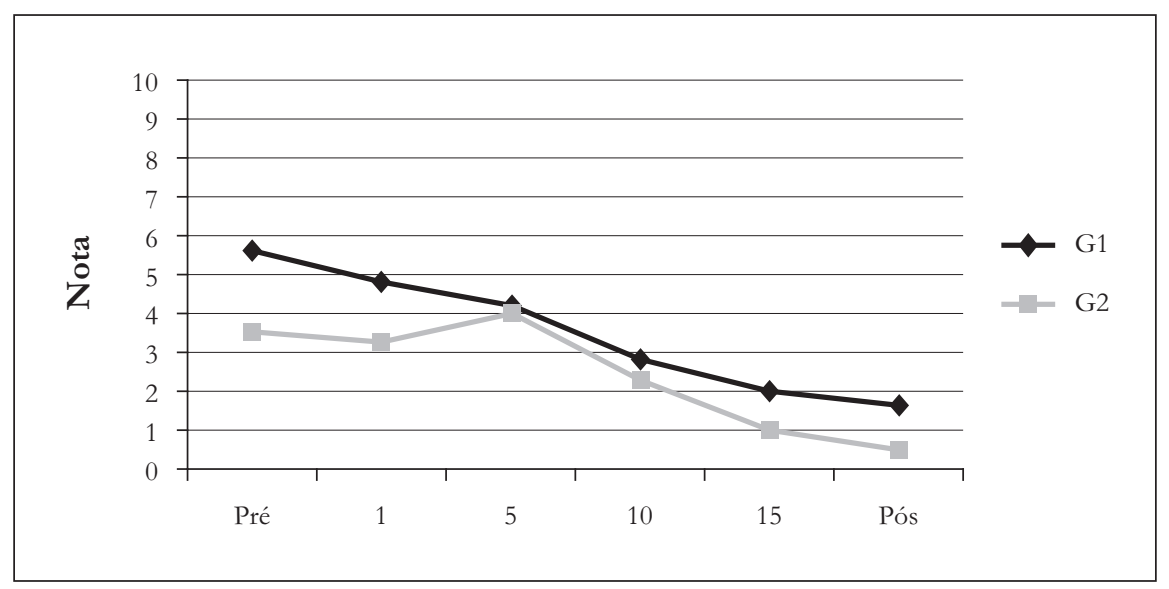

Gráfico1 - Análise comparativa do comportamento da dor entre os Grupos 1 e2 Fonte: Dados da pesquisa. 
Quando analisada a flexibilidade pela distância dedo-solo, o Grupo 1 apresentou uma média inicial de 15,6 \pm 5,89 cm e uma média final de $-0,3 \pm 9,52 \mathrm{~cm}(\neq 16,4 \pm 10,31 \mathrm{~cm}$ entre a avaliação pré e a pós-tratamento) e $\mathrm{p} \leq 0,043$, considerando-se, portanto, esta mudança estatisticamente significativa. Ressalta-se que três dos cinco participantes obtiveram valores negativos, ou seja, ultrapassaram a linha zero, demonstrando acentuada melhora da flexibilidade.

Quando a flexibilidade foi avaliada pela análise do ângulo de flexão do joelho, este grupo apresentou uma média de $33 \pm$ 6,70 graus de flexão em sua avaliação inicial, revelando um evidente encurtamento dos isquiotibiais. Já na avaliação após a intervenção, os sujeitos apresentaram melhora da flexibilidade estatisticamente significativa ( $\mathrm{p} \leq 0,042$ ), sendo que a média de redução do ângulo de flexão foi de 27,8 $\pm 9,44$ graus. Concluímos, portanto, que o programa de mobilização neural mostrou ser eficaz no incremento da flexibilidade da cadeia muscular posterior, tanto na avaliação da distância dedo-solo quanto na avaliação do ângulo de flexão do joelho.

Por sua vez, o Grupo 2 apresentou, em sua avaliação inicial, uma distância dedo-solo de 7,25 \pm 9,46 $\mathrm{cm}$, obtendo aumento da flexibilidade em média de $9 \pm 5,03 \mathrm{~cm}$; no entanto, esta melhora da flexibilidade não pode ser considerada significativa para esta amostra $(\mathrm{p} \leq 0,066)$. Na avaliação do ângulo de flexão do joelho, este grupo apresentou inicialmente média de 23,75 \pm 12,60 graus de flexão do joelho, passando para 9,25 \pm 9,25 graus, com uma diferença de 14,5 \pm 6,95 graus entre a avaliação pré e pós-alongamento muscular, o que indica pequena melhora da flexibilidade, embora esta não possa ser considerada significativa, pois $\mathrm{p} \leq 0,068$.

Realizando a comparação entre os resultados obtidos pós-intervenção e entre as diferenças na avaliação distância dedo-solo do Grupo 1 e do Grupo 2, não houve diferenças estatisticamente significativas ( $\mathrm{p} \leq 0,806)$. Assim sendo, não há como afirmar que, para esta amostra, um método foi mais efetivo em relação ao outro no que se refere à melhora da flexibilidade da cadeia muscular posterior avaliada pela distância dedo-solo (Gráfico 2).

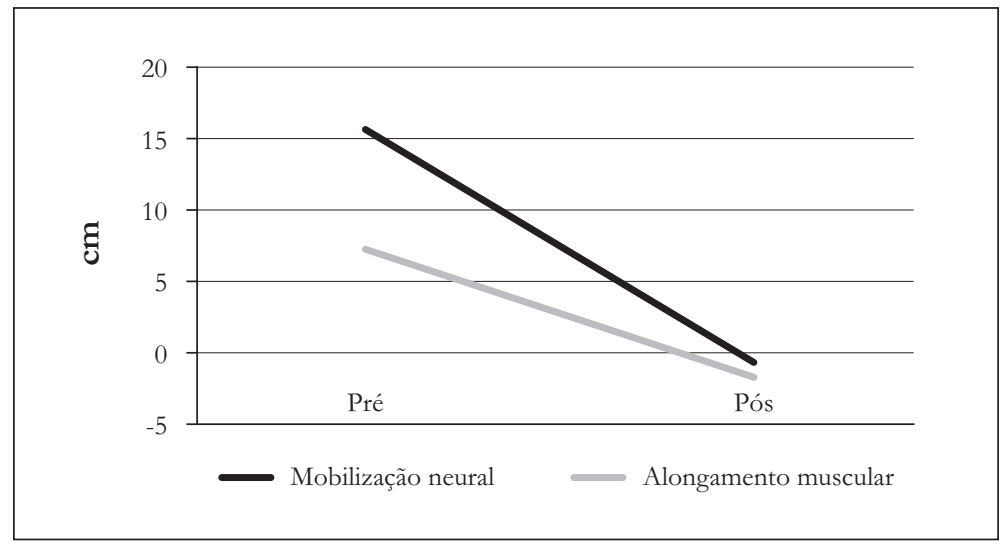

Gráfico 2 - Análise comparativa do teste de distância dedo-solo entre os Grupos 1 e 2

Fonte: Dados da pesquisa.

$\mathrm{Na}$ análise comparativa das avaliações do ângulo de flexão do joelho, não houve diferença estatisticamente significativa entre os resultados obtidos posteriormente à intervenção $(p \leq 0,453)$. É relevante lembrar, no entanto, que embora não havendo grau de significância para o tratamento realizado com o alongamento muscular, é necessário esclarecer que houve melhora na flexibilidade desses sujeitos, como indica abaixo o Gráfico 3.

No que diz respeito à flexibilidade da cadeia muscular posterior, sendo utilizado como instrumento de avaliação a distância dedo-solo e o grau de flexão do joelho, não foi possível referir a superioridade de um método em relação ao outro. Devemos, porém, considerar que ambos os métodos apresentaram melhoras na flexibilidade, embora somente a mobilização neural mostre resultados com grau de significância. 


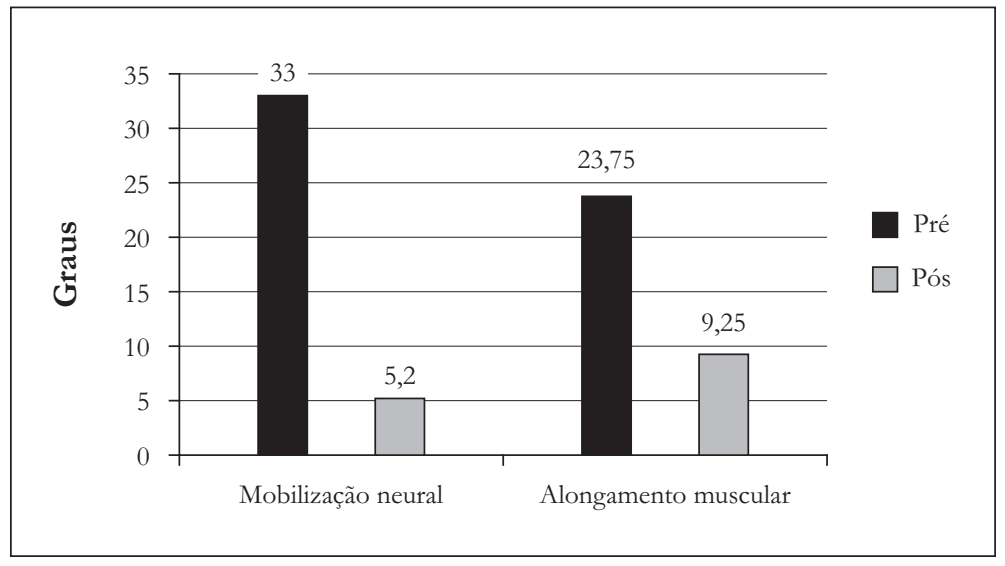

Gráfico 3 - Análise comparativa do ângulo de flexão do joelho entre o Grupo 1 e o Grupo 2

Fonte: Dados da pesquisa.

$\mathrm{Na}$ avaliação das atividades físico-funcionais, realizadas com o emprego do questionário de RollandMorris, o Grupo 1 apresentou média inicial de 15,4 \pm 3,84 pontos, e 3,6 \pm 2,21 ao término da intervenção com a técnica de mobilização neural, com uma diferença média de $11,8 \pm 5,49$ pontos. A análise estatística mostrou que este resultado foi significativo $(\mathrm{p} \leq 0,043)$. Assim, a mobilização do sistema nervoso proporcionou, para esta amostra, melhora significativa na realização das atividades físico-funcionais.

Em contrapartida, no Grupo 2 houve uma média inicial de $11 \pm 2,82$ pontos, reduzindo para 2,25 \pm 2,12 (diferença média de 9,5 \pm 4,94). O programa de alongamento muscular reduziu os escores, melhorando, assim, a capacidade para a realização das atividades físico-funcionais dos sujeitos deste grupo, porém a análise estatística não demonstrou grau de significância $(\mathrm{p} \leq 0,066)$.

Quando comparados os dois grupos, no período pós-intervenção, nesta amostra não houve diferença estatisticamente significativa ( $\mathrm{x} \leq 0,453)$. Estes resultados não apontam para a superioridade de um método em relação ao outro no que diz respeito à melhora para a realização das atividades funcionais. Deve ser lembrado, contudo, que ambos os programas terapêuticos ao serem empregados isoladamente, proporcionaram melhoras nas atividades físico-funcionais, como pode ser constatado no Gráfico 4 a seguir.

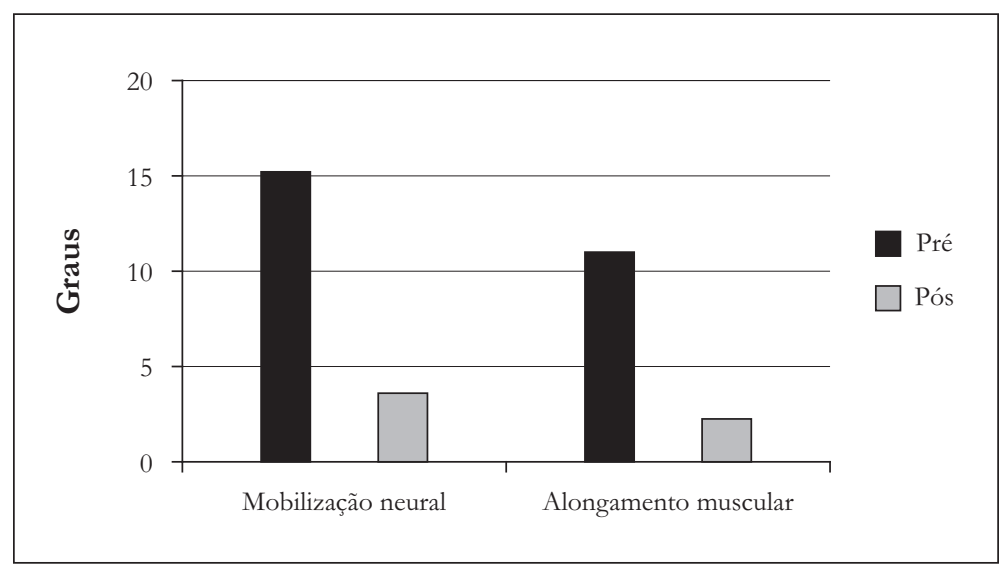

Gráfico 4 - Análise comparativa das atividades funcionais entre o Grupo 1 e o Grupo 2

Fonte: Dados da pesquisa. 


\section{Discussão}

Como já referido, a amostra é constituída em sua maioria por mulheres. Este fato sustenta o que muitos estudos indicam: que a sobrecarga de tarefas ocupacionais e domésticas e o acúmulo de papéis tornam as mulheres mais propensas ao desenvolvimento de patologias músculo-esqueléticas (11). Os resultados encontrados nas avaliações iniciais da tensão neural estão de acordo com conclusões de Hall (12), o qual, em seu estudo com números representativos de pacientes com dores, percebeu que um terço delas apresentava tensão neural.

É importante ressaltar que a tensão neural ocorre em razão de uma patologia que envolve o sistema que circunda a estrutura neural. Esses sistemas são chamados de interfaces mecânicas e movimentam-se independentemente do sistema nervoso (13).

Para que o sistema nervoso periférico seja afetado, não há a necessidade de uma lesão direta. Poderia muito bem ser uma lesão secundária como resultado de sangue e edema de uma interface lesada ou uma alteração na forma da interface (9). Anomalias nervosas, ou anomalias de tecidos interfacetários, possibilitam a predisposição do sistema à lesão. A lesão implica alteração das funções do nervo, por conseguinte a alteração da condução elétrica acarreta distúrbios sensoriais (dor e parestesias), distúrbios motores (distonias) e fraqueza autonômica (vasomotores e pilomotores) (14).

Segundo Butler (9), o sistema nervoso está certamente envolvido, direta ou indiretamente, em todos os problemas do paciente. Ele pode estar lesado e ser uma fonte de sintomas ou, mesmo quando não lesado, transmitir impulsos aferentes de estruturas não neurais e sinais eferentes de respostas. Por exemplo: um espasmo muscular que pode ter como resultado a dor lombar.

Uma lesão nervosa, portanto, gera alterações em suas propriedades mecânicas (movimento e elasticidade) e fisiológicas (alterando sua neurodinâmica), que, por sua vez, sustentam ou agravam a lesão. Tais lesões podem derivar para disfunções nas estruturas que recebem sua inervação. Como consequência, estruturas músculo-esqueléticas podem restar comprometidas em uma disfunção de origem neural (15).

Nesse sentido, é importante referir que os testes de tensão neural têm importância fundamental na avaliação fisioterapêutica das síndromes dolorosas da coluna lombar, e que estes testes são aplicados com o objetivo de quantificar e qualificar os desvios físico-funcionais (16). A avaliação da tensão neural tornase, portanto, fundamental para o tratamento de pacientes com disfunções osteomusculares. Dessa forma, a aplicação da tensão neural contribui para a formulação de um diagnóstico mais preciso e, consequentemente, influencia no sucesso do tratamento (17).

Em relação ao quadro álgico, pode ser constatado que o programa de mobilização neural reduziu de forma significativa a dor pela avaliação da EAV. Por sua vez, o programa de alongamento muscular (Grupo 2) não reduziu de forma significativa a dor dos sujeitos que receberam a intervenção com base neste programa de tratamento. Este fato pode estar relacionado ao número reduzido da amostra.

No que diz respeito à mobilização neural, tanto neste estudo como no apresentado por Boeing (18) e Caraviello et al. (19), esta técnica mostrou-se eficiente na redução da dor em pacientes portadores de lombalgia crônica. Santos (20) obteve resultado similar ao aplicar técnicas de mobilização neural em um sujeito com câimbra do escrivão. Guelfi (21) observou resultados significativos na redução da dor empregando a mobilização neural em um paciente com siringomiela.

Por outro lado, a redução da dor lombar ocasionada pelo programa de alongamento muscular está relacionada ao alívio da tensão muscular, pois quando uma musculatura encontra-se em tensão, pode ocasionar pequenos deslizamentos nas vértebras e desgastá-las, pressionando os nervos e provocando dor na coluna (22).

O alongamento muscular é fundamental para o movimento. Dessa forma, a flexibilidade desempenha um papel importante para a boa execução das atividades diárias, favorecendo a prática e o desempenho do movimento especializado (23).

A baixa flexibilidade muscular resulta em amplitude articular reduzida em várias tarefas diárias, por conseguinte se terá, normalmente, rigidez articular e limitação grave do movimento, impedindo a realização dessas atividades desvinculadas da dor. A flexibilidade da coluna lombar fornece uma vantagem mecânica para a função e para a eficiência do movimento (24). 
O que se observa, quando é avaliada a flexibilidade muscular, é que esta amostra possui um encurtamento de isquiotibiais bastante significativo, como ficou demonstrado pela avaliação do ângulo de flexão do joelho (22). A rigidez dos músculos isquiotibiais é compensada com frequência pela movimentação da coluna lombar, que irá exercer mais carga sobre a coluna, ocasionando menor mobilidade no quadril e contribuindo assim para a lombalgia (24).

Outro fator importante é que, com o aumento da flexibilidade muscular, as atividades de vida diária (AVDs) podem ser executadas com maior amplitude de movimento, maior força, mais rapidamente, mais facilmente, com maior fluência e de modo mais eficaz. Ressalta-se ainda que a falta de flexibilidade constitui fator limitante ao desempenho das funções diárias, sendo considerada um facilitador de lesões musculares (25).

Até o momento, porém, a literatura não traz comprovações científicas de que o treinamento isolado da flexibilidade proporciona resultados significativos para a estabilização do tronco e alívio das dores lombares, uma vez que um dos fatores responsáveis pela dor lombar é a hipotrofia dos músculos estabilizadores do tronco, que geram fraqueza e fragilidade da coluna lombar (26).

O aumento da flexibilidade quando utilizada a mobilização neural está relacionado à restauração do movimento e à elasticidade do sistema nervoso, promovendo, assim, o retorno às suas funções normais - partindo do princípio fundamental segundo o qual tanto o sistema nervoso central quanto o periférico devem ser considerados como unitários, uma vez que ele forma um trato tecidual contínuo, estando ligado quimicamente, eletricamente e pela continuidade dos tecidos conjuntivos (12).

Se o sistema nervoso fosse considerado um órgão em vez de uma estrutura multissegmentada, isto levaria a um entendimento mais aprofundado do sistema e das consequências patomecânicas e patofisiológicas de alterar sua mecânica. Umas das maiores implicações de vê-lo como um órgão é que, se for constatada qualquer alteração em alguma parte do sistema, isto trará repercussões para todo o sistema (15).

Devemos lembrar que um dos objetivos da mobilização neural é restaurar ângulos em articulações com amplitude de movimento limitada pela dor (27), o que estamos demonstrando no presente estudo.

Pelos resultados apresentados, não há como afirmar que a mobilização neural seja mais eficiente que o alongamento muscular para a melhora da flexibilidade. Fonteque et al. (28), no entanto, em estudo semelhante, compararam a mobilização do sistema nervoso com o alongamento passivo e constataram a maior eficácia da mobilização neural no ganho de amplitude de movimento do quadril em relação ao alongamento passivo.

Diante da análise dos resultados, pode-se observar grau de incapacidade elevado, pois a dor crônica ocasiona limitações funcionais nas atividades de vida diária e de vida prática, além de restrição na participação do indivíduo na sociedade (28).

Jesus (24) aplicou a técnica de mobilização neural com oscilações lentas em pacientes com tensão neural e verificou aumento da flexibilidade dos isquiotibiais, concluindo que a mobilização do nervo interfere no fluxo exoplasmático, podendo ser esperada a melhora da função neural.

Assim, a melhora das atividades funcionais tem relação direta com a redução da dor e com o aumento da flexibilidade, uma vez que indivíduos que apresentam baixa flexibilidade muscular, resultando em amplitude articular reduzida em várias tarefas diárias, por conseguinte terão normalmente rigidez articular e limitação grave do movimento, impedindo a realização dessas atividades desvinculadas da dor (29).

\section{Considerações finais}

A dor lombar repercute diretamente na qualidade de vida dos sujeitos, gerando diversos graus de incapacidade física, o que ficou demonstrado neste estudo, quando aplicado o questionário de RollandMorris. Observou-se um significativo impacto da dor nas atividades funcionais e isto pode ter relação direta com o tempo de instalação da disfunção e o quadro álgico apresentado pelos indivíduos, como foi destacado na pesquisa.

Pela análise dos resultados obtidos, tanto o programa de mobilização neural quanto o programa de alongamento muscular revelaram melhoras na execução das atividades funcionais, na flexibilidade da cadeia muscular posterior e na redução do quadro álgico. Ressalta-se, porém, que somente o programa de mobilização 
neural obteve melhora estatisticamente significativa. Não foi possível, entretanto, destacar a maior eficácia de um tratamento em relação ao outro, o que pode ser explicado pelo fato de a amostra ser de um número reduzido de participantes, considerando-se esta a maior fonte de limitação do estudo.

Neste propósito faz-se necessário lembrar que o tratamento fisioterapêutico não deve se limitar a uma única técnica, mas deve atender às mais diferentes necessidades do paciente. Nesse objetivo é importante uma abordagem terapêutica ampla e baseada no emprego das mais diversas técnicas.

Por fim, destaca-se que este estudo não teve o objetivo de sanar todas as dúvidas que giram em torno deste tema. Espera-se, todavia, auxiliar os profissionais na elaboração de um protocolo terapêutico e estimular o desenvolvimento de futuras pesquisas que possam trazer comprovações científicas sobre a técnica de mobilização neural.

\section{Referências}

1. Cecin HA. Proposição de uma reserva anatomofuncional, no canal raquidiano, como fator interferente na fisiopatologia das lombalgias e lombociatalgias mecânico-degenerativas. Rev Assoc Med Bras. 1997;43(4):295-310.

2. Canavan PK. Reabilitação em medicina esportiva: um guia abrangente. São Paulo: Manole; 2001.

3. da Silva MC, Fassa ACG, Valle NCJ. Dor lombar crônica em uma população adulta do sul do Brasil: prevalência e fatores associados. Cad. Saúde Pública [periódico online] 2004 [citado 25 set. 2007];20(2). Disponível em: http:// www.scielo.br/scielo.php?script=sci_arttext\&pid=S0102-311X2004000200005 \&lng= pt\&nrm=iso

4. Silva CN, Silva AT, Gervásio FM. Prevalência e aplicação da classificação de Mckenzie para lombalgia em funcionários do Centro Universitário UniEvangélica. [citado 21 mar. 2008]. Disponível em: http:/ /www.unievangelica. edu.br/noticias/969/fisioterapia/IC\%2009.02.06\%20subp2.pdf

5. Cox JM. Dor lombar: mecanismos, diagnósticos e tratamento. São Paulo: Manole; 2002.

6. Mendes R. Patologia do trabalho. São Paulo: Atheneu; 2005.

7. Bisschop P, Ooteghem P van. Dor lombar: evidência clínica para conceito dural. [citado 12 out. 2007]. Disponível em: http://www.fisioterapiasalgado.com.br/visualiza.asp?id=403

8. Costardi CHZ, Baptistella RCF, Gomes AC, Júnior DB, Claret DC. Influência das técnicas de mobilização neural e articular na qualidade de vida de pacientes com dor lombar crônica. [citado 20 fev. 2008]. Disponível em: www.afb.org.br/intercobraf/revista/terapiamanual/autor/CHRISTINA\%20HELENA\%20ZANOTTO $\% 20$ COSTARDI $\% 20-\% 20558 . p d f$

9. Butler DS. Mobilização do sistema nervoso. São Paulo: Manole; 2003.

10. Kisner C, Colby LA. Exercícios terapêuticos: fundamentos e técnicas. 3a ed. São Paulo: Manole; 1998.

11. Lancman S. Saúde, trabalho e terapia ocupacional. São Paulo: Roca; 2004.

12. Hall T. Mobilização neural: novo conceito manipulativo. Revista do Conselho Federal de Fisioterapia e Terapia Ocupacional [periódico online] 2004 [citado 15 maio 2008];21(2):20. Disponível em: http://www.brasilmedicina. $\mathrm{com} /$ noticias $/$ pgdetalhe.asp?Cod=736\&Area $=3$

13. Guelfi MD. A influência da mobilização do sistema nervoso em um indivíduo portador de siringomiela. Rev Terapia Manual. 2004;2(4):158-61.

14. Smaniotto ICG, Fonteque MA. A influência da mobilização do sistema nervoso na amplitude de movimento da flexão do quadril. Rev Terapia Manual. 2004;2(4):154-7.

15. Marinzeck S. Mobilização neural: aspectos gerais. [citado 20 mar. 2007]. Disponível em: http://www.terapiamanual. com.br/site/noticias/arquivos/200912101725220.artigo_7.pdf 
16. Bracht MA. Estudo comparativo entre os testes Slumpe Laségue em pacientes portadores de síndromes dolorosas da coluna lombar. Rev Terapia Manual. 2003;2(2):46-51.

17. Beleski, RC. Verificação da presença de tensão neural nas cervicobrauialgias através dos testes de tensão neural para nervo mediano e radial. Rev Terapia Manual. 2004;2(4):182-5.

18. Boeing M. Análise da eficácia de técnicas de mobilização neural para pacientes com ombociatalgia [trabalho de conclusão]. Cascavel: Universidade Estadual do Oeste do Paraná. 2004.

19. Caraviello EZ, Wasserstein S, Chamlian TR, Masiero D. Avaliação da dor e função de pacientes com lombalgia tratados com um programa de escola de coluna. Acta Fisiatr. 2005;12(1):11-14.

20. Santos VR. A influência da mobilização do sistema nervoso na câimbra do escrivão. Rev Terapia Manual. 2004;2(4):166-71.

21. Guelfi MD. A influência da mobilização do sistema nervoso em um indivíduo portador de siringomiela. Rev Terapia Manual. 2004;2(4):158-61.

22. Achour Jr. A. Bases para exercícios de alongamento: relacionado com saúde e no desempenho atlético. 2a ed. São Paulo: Phorte; 1999.

23. Jesus CS. A mobilização do sistema nervoso e seus efeitos no alongamento da musculatura ísquiotibial. Rev Terapia Manual. 2004;2(4):162-5.

24. Alter MJ. Ciência da flexibilidade. 2a ed. Porto Alegre: Artmed; 1999.

25. Bertolla F, Baroni BM, Leal Jr. ECP, Oltramari JD. Efeito de um programa de treinamento utilizando o método Pilates ${ }^{\circledR}$ na flexibilidade de atletas juvenis de futsal. Rev Bras Med Esporte. [periódico online] 2007 [citado 12 maio 2008];13(4). Disponível em: http://www.scielo.br/scielo.php?script=sci_arttext\&pid=S1517-86922007000400002

26. Costa D, Palma A. O efeito do treinamento contra resistência na Síndrome da dor lombar. Rev Port Cien Desp. 2004;2:224-34.

27. Carvalho RK, de Macedo ACB. Avaliação da eficácia da aplicação de mobilização neural em digitadores bancários. Rev Terapia Manual. 2005;3(13):490-4.

28. Fonteque MA, Petry CO, Salgado ASI, Pacheco MTTT. Estudo da eficácia da mobilização do sistema nervoso e do alongamento passivo para ganho de amplitude de movimento de flexão de quadril. [citado 3 jun. 2008]. Disponível em: http://www.inicepg.univap.br/INIC_2005/epg/EPG4/EPG4-16\%20ok.pdf

29. Sampaio RF, Mancini MC, Gonçalves GGP, Bittencourt NFN, Miranda AD, Fonseca ST. Aplicação da classificação internacional de funcionalidade, incapacidade e saúde (cif) na prática clínica do fisioterapeuta. Rev Bras Fisioter. 2005;9(2):126-9.

30. Toscano JJO, do Egypto EP. A influência do sedentarismo na prevalência de lombalgia. Rev Bras Med Esporte. 2001;7(4). [citado 25 set. 2007]. Disponível em: http://www.scielo.br/scielo.php?script=sci_arttext\&pid=S1517$86922001000400004 \& \operatorname{lng}=$ pt\&nrm $=$ iso

Recebido: 26/02/2009

Received: 02/26/2009

Aprovado: $13 / 05 / 2010$

Approved: 05/13/2010 